\title{
Capabilities of Tomosynthesis in Diagnosing Chest Diseases and Injuries
}

\author{
DOI: $10.17691 / \mathrm{stm} 2016.8 .2 .08$
}

Received October 10, 2015

\begin{abstract}
V.A. Nechaev, Radiologist, Radiology Department';
A.Yu. Vasiliev, MD, DSc, Corresponding Member of the Russian Academy of Sciences, Professor, Department of Radiology ${ }^{2}$

${ }^{1}$ City Clinical Hospital No.4, 25 Pavlovskaya St., Moscow, 115093, Russian Federation;

${ }^{2}$ Moscow State University of Medicine and Dentistry named after A.I. Evdokimov, 20/1 Delegatskaya St., Moscow, 127473, Russian Federation
\end{abstract}

The aim of the investigation is to assess the capabilities of tomosynthesis (TS) in diagnosing chest diseases and injuries.

Materials and Methods. One hundred and twenty eight patients admitted to the reception ward of Moscow City Hospital with various complaints were examined. All the patients underwent digital radiography (DR) of the chest as clinically indicated. When changes were revealed, chest TS was performed in a standing or lying position. To clarify the nature of lung tissue lesions, 44 patients were examined by multislice computed tomography (MSCT). According to DR findings, the patients were divided into five groups depending on the key $\mathrm{X}$-ray syndrome: with the signs of lung consolidation $(n=29)$, nodules $(n=28)$, destructive lung lesions $(n=24)$, disseminated process $(n=24)$ and urgent conditions $(n=23)$.

Results. In some cases TS has allowed exclusion of a pathological process and identifying additional changes, namely, the signs of localized tinyfocal dissemination, lung tissue destruction, "bronchial amputation" and "air bronchogram" symptom, nodules, rib injuries and others. TS improves the abilities of X-ray radiography, it is superior to DR in the detection of many X-ray semiotic signs and can be used to provide additional diagnostic accuracy.

Conclusion. TS is inferior to MSCT in diagnosing chest diseases and injuries, however, in $67.2 \%$ of observed cases it allows acquisition of additional information. In $39.8 \%$ of cases this information has proved to be clinically significant and influenced further management of these patients.

Key words: tomosynthesis; digital radiography; multislice computed tomography; chest diseases and injuries.

The proportion of respiratory system pathology in total morbidity pattern of Russian population amounts to $24.2 \%[1,2]$. At the initial stage standard digital radiography (DR) is used as a radiological method to diagnose lung diseases. However, if pathological changes are revealed additional examination by means of multislice computed tomography (MSCT) is very often required to specify their character [3].

In recent years, tomosynthesis (TS), an X-ray technique that generates a number of cross-sectional images of the patient's examined area from a single pass of the X-ray tube, has attracted great attention in thoracic diagnosis. It improves the detection and characterization of pathologic chest lesions revealed by DR but has a relatively low dose of radiation [4].

Despite the TS benefits in diagnosing various lung diseases, so far its place in the algorithm of such patients' examination has not been completely determined [3-5].

The aim of the investigation is to assess the capabilities of tomosynthesis in diagnosing chest diseases and injuries.
Materials and Methods. One hundred and twenty eight patients aged 18 to 82 years (84 male $(65.8 \%)$ and $44(34.2 \%)$ female) were examined at the admission department of Moscow City Hospital. After clinical and laboratory examination all the patients underwent chest DR as clinically indicated. In order to specify the character of pathological changes TS was performed collecting frontal view in a vertical $(n=87 ; 68 \%)$ and lying position $(n=41 ; 32 \%)$ with the following physicotechnical parameters: focal length: $130 \mathrm{~cm}$, tube movement angle: 25 and $30^{\circ}$ (standing and lying positions, respectively), central slice height: $150 \mathrm{~mm}$, range: $260 \mathrm{~mm}$, slice separation: $5 \mathrm{~mm}$.

The study complies with the Declaration of Helsinki (adopted in June 1964 (Helsinki, Finland) and revised in October 2000 (Edinburgh, Scotland)) and was performed following approval by the Ethics Committee of Moscow State University of Medicine and Dentistry named after A.I. Evdokimov. Written informed consent was obtained from every patient.

In TS process a definite number of images of the examined area is acquired at a single pass of the X-ray

For contacts: Valentin A. Nechaev, e-mail: dfkz2005@gmail.com 
tube. The way the images are generated resembles that of linear tomography, however in TS a series of "raw" images is acquired which undergo further computed processing. To eliminate image blurriness, various reconstruction algorithms are applied, "shift-and-add" and "filtered back projection" being the most commonly used methods among them. During chest organ examination approximately 53 frontal tomograms are produced.

To clarify the character of lesions with an unclear X-ray pattern MSCT was performed according to standard protocol $(n=44 ; 34.4 \%)$. DR and TS were carried out using FDR AcSelerate $200 \mathrm{X}$-ray system (Fujifilm, Japan), for MSCT Aquilion Prime 160 apparatus (Toshiba, Japan) was used.

Results and Discussion. According to DR findings, all patients were divided into 5 groups depending on the key X-ray semiotic symptom:

group 1 - lung consolidation $(n=29 ; 22.66 \%)$;

group 2 - nodules $(n=28 ; 21.88 \%)$; group 3 - lung and skeletal tissue destruction $(n=24$; $18.75 \%)$;

group 4 - disseminated process $(n=24 ; 18.75 \%)$;

group 5 - urgent conditions ( $n=23 ; 17.97 \%)$.

According to DR data, in 26 patients of group 1 the signs of lung consolidation were detected. On performing TS the presence of the key X-ray semeiotic sign was confirmed in 23 cases $(79.3 \%)$, in 5 of them it was possible to make segmental localization of pathological process more accurate, in 4 cases opacity structure was clarified.

However, in 6 patients no lung tissue consolidation was detected. First of all this could be due to the presence of summation effect that standard X-ray pictures demonstrated. The changed lung pattern $(n=4)$, fibrotic changes $(n=1)$ and a combination of intensified pulmonary pattern, fibrosis and mediastinal fat tissue proliferation $(n=1)$ were taken for opacity symptoms on the films (Figure 1).

Additionally, the changes revealed by TS influenced
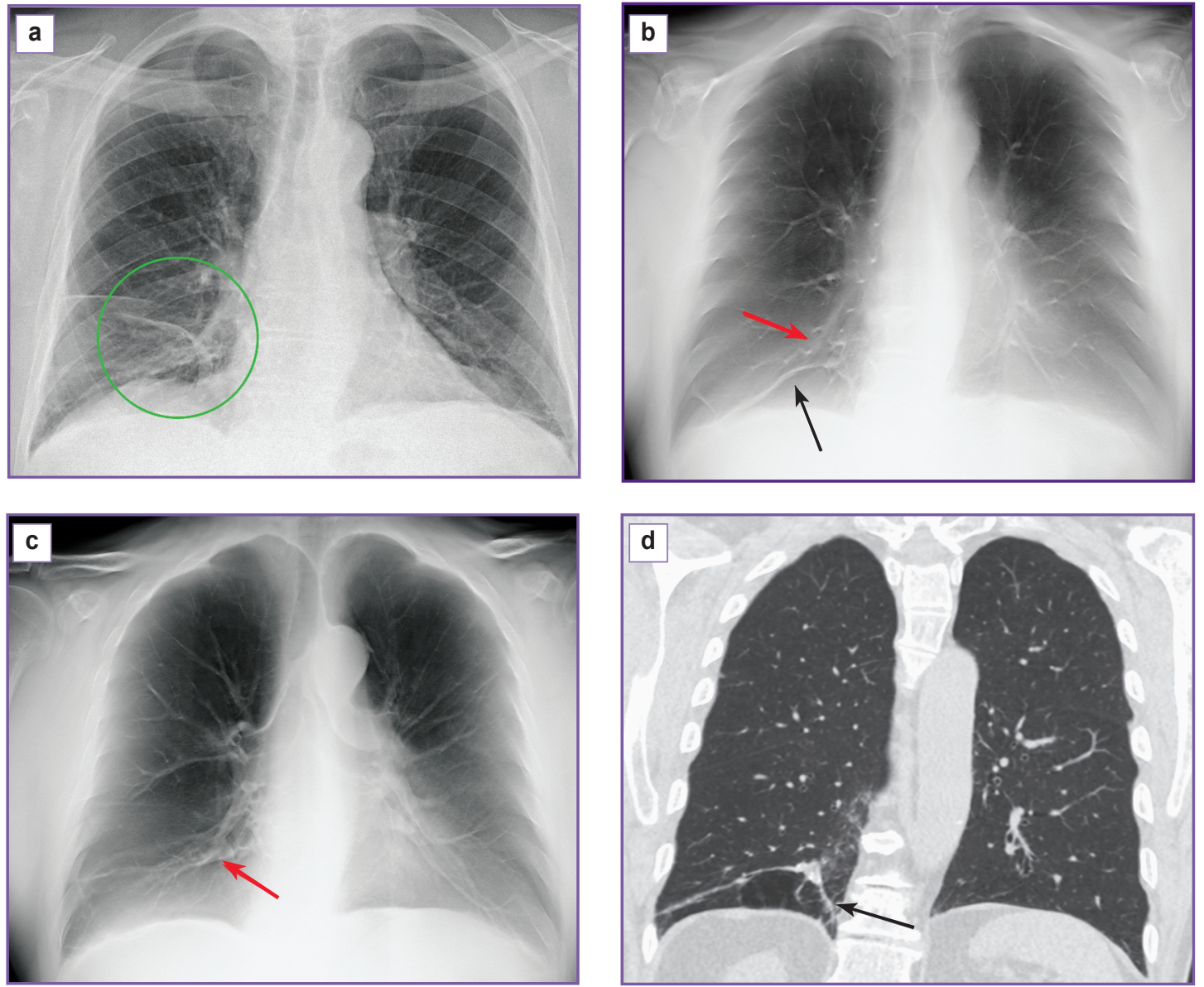

Figure 1. A frontal digital chest radiograph (a), chest tomograms (b), (c) and a computed chest tomogram (d) in the coronal plane. The radiograph demonstrates the reduction of lung tissue transparency (green circle) within the right lower lung fields. The tomograms show accentuated lung pattern (red arrows) and fibrotic changes (black arrows) in the region of suspected infiltrative changes of the lung tissue, which is confirmed by the computed tomogram 
further therapeutic approach. So, the signs of destructive changes $(n=4)$, "air bronchogram" symptom $(n=5)$, lung parenchyma fibrosis $(n=6)$, tiny-focal dissemination and "bronchial amputation" symptom $(n=2)$ were detected on the background of lung consolidation (Figure 2).

In tomograms of 18 patients from this group (62.1\%) the signs of dynamic blurriness were noted. In 5 cases they significantly affected the image quality reducing its diagnostic value.

In one case a metal structure was detected in the examined area which was the reason for marked artefacts on different slices.

On MSCT examination ( $n=16 ; 55.2 \%)$ the leading X-ray semiotic sign was confirmed in 12 out of 16 patients $(75 \%)$ while it was excluded in 2 patients and the signs of lung tissue consolidation unnoted in digital tomograms (TS) were detected in 2 cases. False-positive and false-negative results were mostly associated with the presence of breathing artifacts in the tomograms. In all cases the missed or "false" changes were located in supradiaphragmatic areas on the right or on the left behind the heart shadow.

Additionally, MSCT examination revealed the signs of hydrothorax $(n=8)$, panlobular and paraseptal emphysema $(n=4)$, single calcifications in the lung parenchyma $(n=4)$, ground-glass opacities, calcified intrathoracic lymph nodes, calcified coronary vessels and periapical adhesions $(n=3)$. In 2 cases hydropericardium signs and traction bronchiectases were noted. Besides,

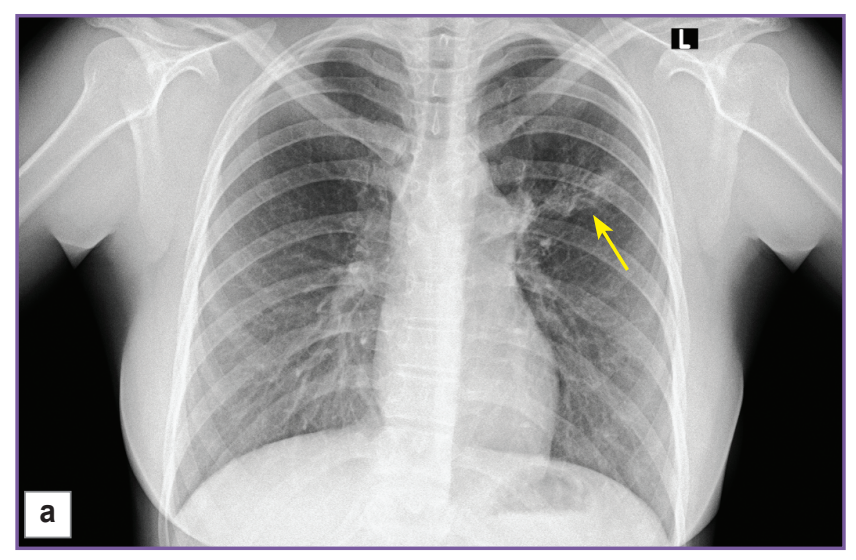

Figure 2. A frontal digital chest radiograph (a) and chest tomograms in the coronal plane (b), (c). The radiograph shows heterogeneous parenchymatous lung infiltration (yellow arrow) within the left upper lung fields. The tomograms demonstrate the signs of tiny-focal dissemination (green arrows) and "air bronchogram" symptom (red arrow) the signs of bronchiolitis, lung tissue destruction, fibrotic and hypoventilatory changes and a central lung mass $(n=1)$ were detected.

Group 2 included 28 patients with rounded nodular shadows suspected or revealed on standard radiographs.

TS examination confirmed the presence of pulmonary nodules (27 in number) in 18 patients $(64.3 \%)$, while pseudolesions (12) were noticed in 10 patients. Specific lung pattern (4), fibrotic changes (2), first rib calcification in the area of costochondral junction (2), a mass in the soft chest tissues (2), consolidated fracture (1) and infiltrative changes in the lung tissue (1) were taken for masses (Figure 3).

TS as compared to DR allowed detecting 23 nodules additionally in 12 cases $(42.9 \%)$. There were also revealed the signs of localized fibrosis (12), calcified intrathoracic lymph nodes (6), calcified areas in the lung parenchyma (5), the signs of aortic sclerosis (4), pulmonary bulla (1), pleuro-apical adhesions (1), cavity of disintegration (1), tiny-focal dissemination against the background of accentuated and distorted lung pattern (1) and Rigler symptom (1) (Figure 4).

In 6 cases $(21.4 \%)$ motion and breathing artefacts were noted on tomograms, which reduced diagnostic accuracy and detectability of pathological changes.

MSCT was performed in 9 patients (32.1\%). In 8 of them presence of 23 masses and 2 pseudolesions was confirmed. Additionally, 10 more nodules visualized
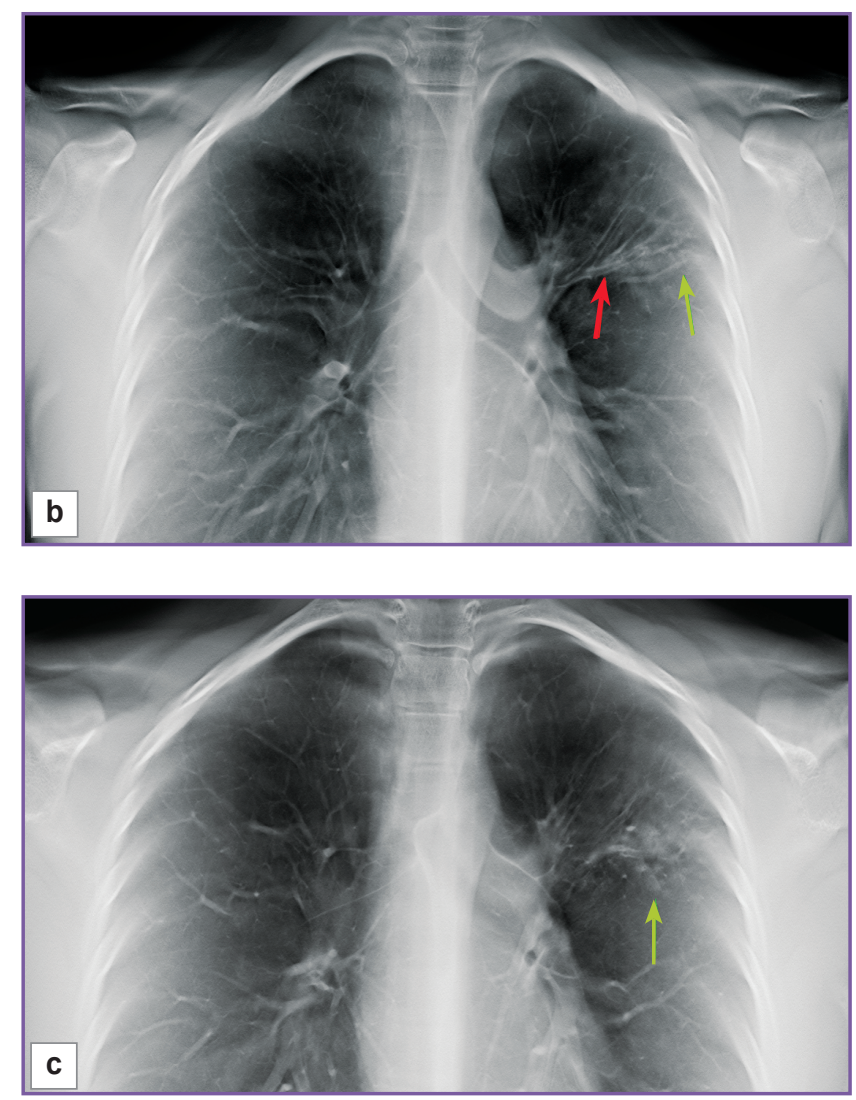

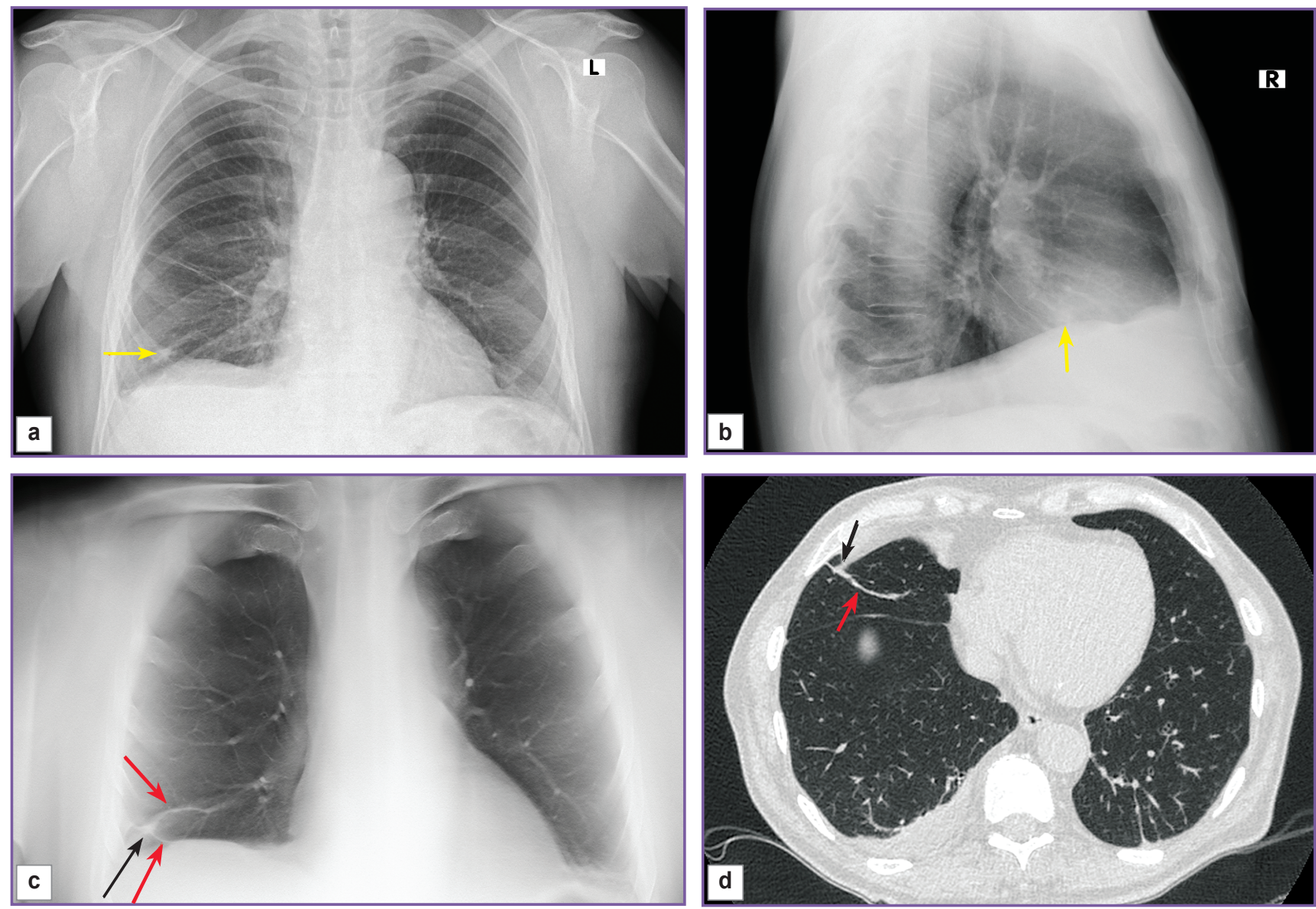

Figure 3. Digital chest radiographs in frontal (a) and lateral (b) projection, a chest tomogram in the coronal plane (c), and a computed chest tomogram in the axial plane (d). The radiographs show ill-defined irregular-shaped nodular shadow of moderate intensity $5 \times 7 \mathrm{~mm}$ in size in $\mathrm{S} 5$ segment of the right lung (yellow arrows). The digital and computed tomograms demonstrate high-intensity shadow (black arrows) passing into the bands of fibrous tissue (red arrows) in the area of suspected nodule

on neither DR nor TS were detected. Besides, MSCT examination revealed enlarged intrathoracic lymph nodes (6), hydrothorax (3), ground-glass opacities and lung tissue consolidations (2), calcified coronary vessels (2), the signs of bronchiolitis (1), traction bronchiectasis (1), bronchial wall calcification (1), centrilobular emphysema (1) and "air bronchogram" symptom (1).

Group 3 included 24 patients whose digital radiographs demonstrated the signs of lung and skeletal tissue destruction.

On performing TS, the changes were confirmed in all cases, at the same time 13 patients' tomograms (54.2\%) showed more cavities than radiographs.

As in other subgroups, TS additionally revealed the signs of tiny-focal dissemination, "air bronchogram" symptom, fibrotic changes $(n=5)$, nodules $(n=3)$ and consolidated rib fractures $(n=2)$. In 2 cases a segmental bronchus coming to the cavity (the drainage bronchus) was defined.

In 9 TS cases motion and breathing artefacts were noted, which reduced the quality of additionally acquired images.

To make the character of pathological changes more accurate, 11 patients underwent MSCT (45.8\%). Destructive changes of the lung tissue were confirmed in 9 patients, in 5 cases there were revealed more cavities of disintegration, while this process was excluded in one patient (Figure 5).

Group 4 included 24 patients with disseminated process in the lungs. According to DR data, the signs of extensive disseminated pulmonary process were detected in 14 cases $(58.3 \%)$ and in 10 cases $(41.7 \%)$ the process was localized.

According to TS findings, the changes were confirmed in 23 patients (95.8\%). However in one case the presence of localized disseminated process was excluded: fibrotic changes in the right lung apex imitated a pathological process due to summation effect on digital radiographs.

In 13 cases $(54.2 \%)$ a greater number of nodular changes undetected on digital radiographs were 

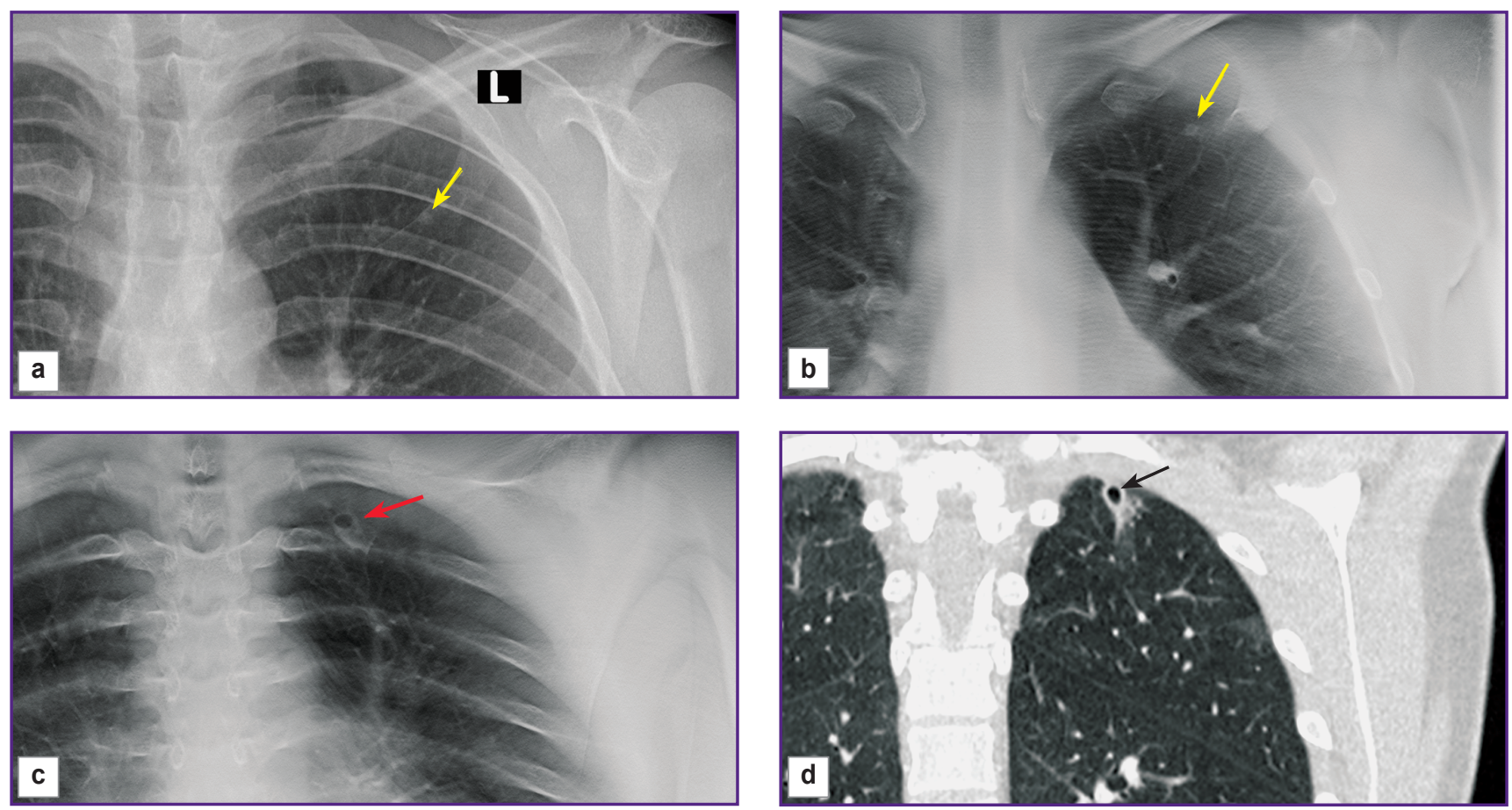

Figure 4. A digital chest radiograph in frontal projection (a), chest tomograms (b), (c) and a computed chest tomogram (d) in the coronal plane. The radiograph shows a rounded ill-defined irregular-shaped nodular shadow of moderate intensity $4 \mathrm{~mm}$ in diameter in the left upper lung field (yellow arrow). The tomograms confirm the presence of the above-described nodule in S3 segment of the left lung (yellow arrow). Additionally, a heterogeneous (due to the presence of the cavity) irregular-shaped mass $7 \times 10 \mathrm{~mm}$ in size is seen in $\mathrm{S} 1+2$ segment on the left (red arrow), which is also evident on the computed tomogram (black arrow)
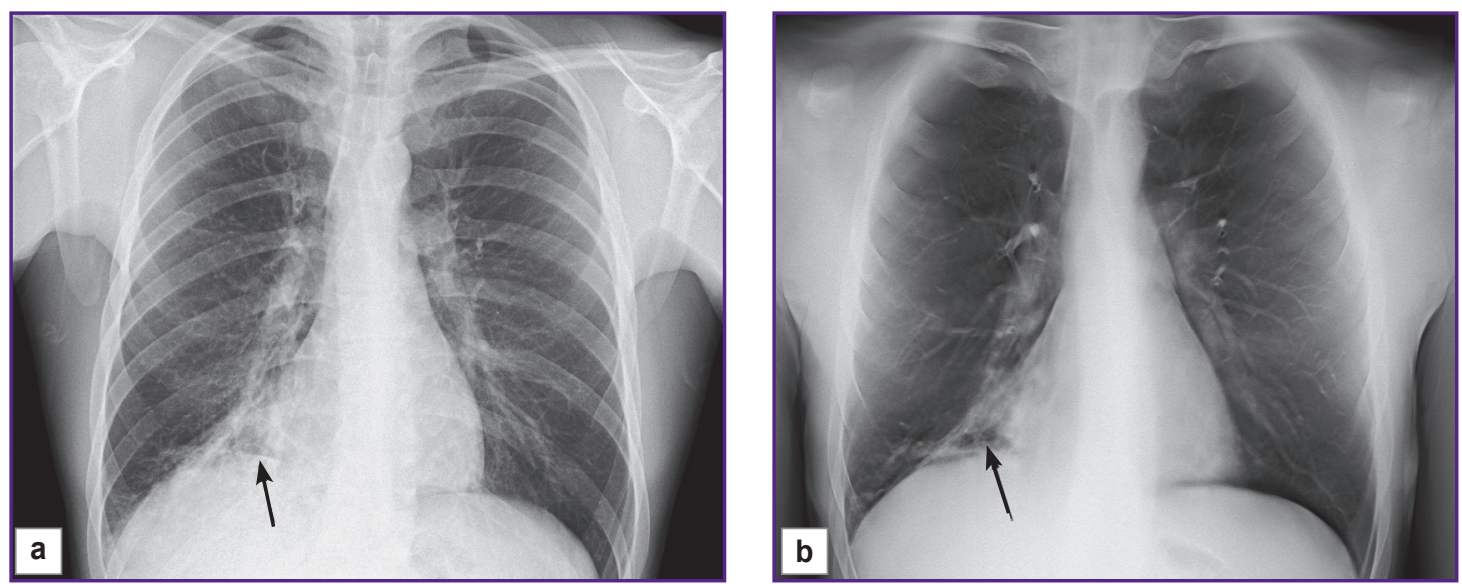

Figure 5. A digital chest radiograph in frontal projection (a), a chest tomogram (b) and a computed chest tomogram (c) in the coronal plane. The digital radiograph and the tomogram show a cavity against the background of localized lung tissue opacity (black arrows). The computed tomogram demonstrates the area of lung tissue consolidation. The intact lung tissue circumscribed by the cupula of the diaphragm on one side and by infiltration on the other imitates destructive changes (red arrow)

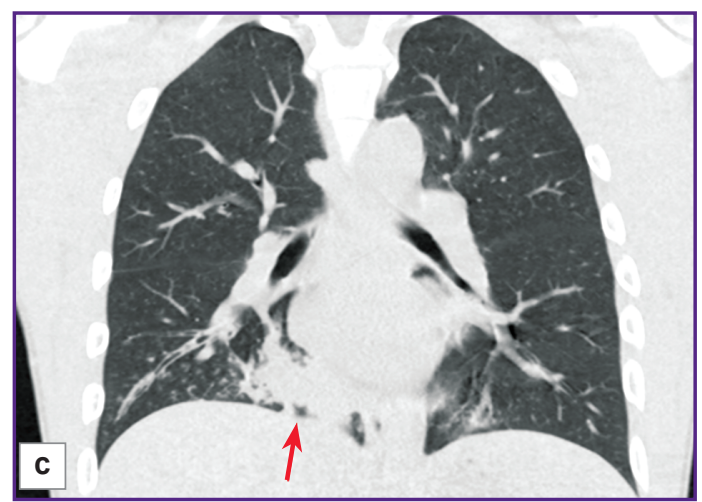



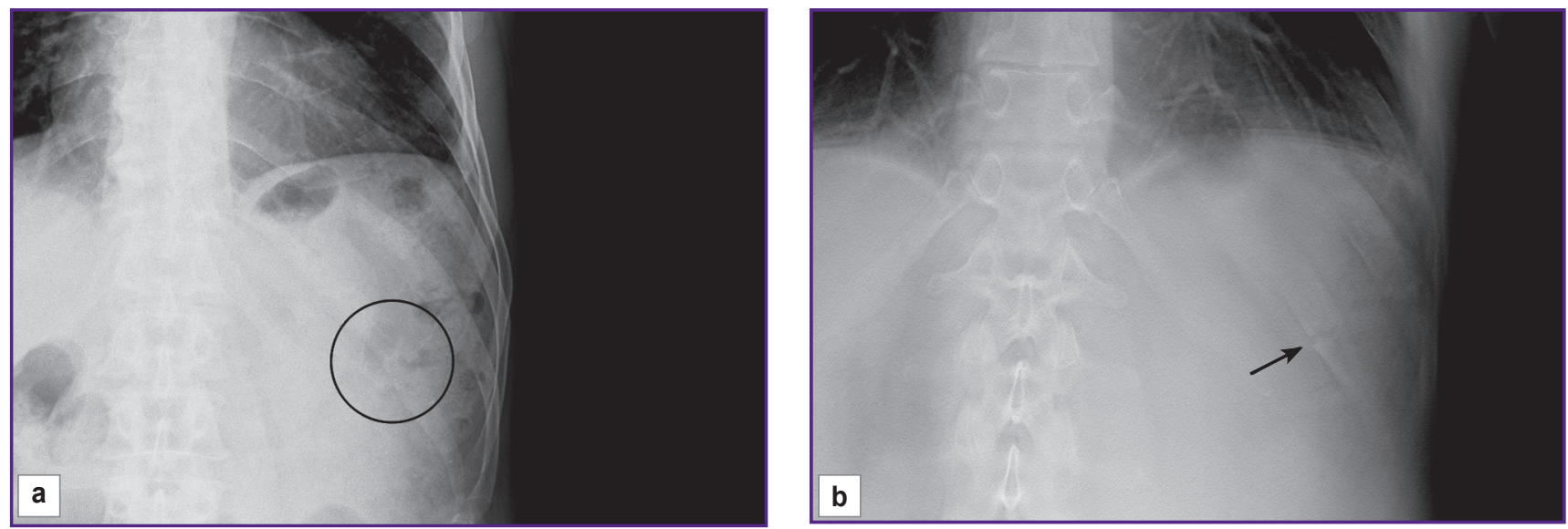

Figure 6. A digital chest radiograph (a) and a chest tomogram (b) in frontal plane. The tomogram shows a transverse fracture of rib XI on the left along the scapular line with a diastasis between the fragments up to $2 \mathrm{~mm}$ (black arrow), which has not been visualized on the radiograph due to accumulation of gases in the intestine (black circle)

defined visually. The signs of bronchiectasis, aortic arch atherosclerosis (three cases) and "air bronchogram" symptom (one patient) were also visualized.

On performing chest MSCT $(n=4)$, presence of disseminated process was confirmed in all the cases and at the same time there was obtained additional information such as the signs of bronchiolitis, groundglass opacities and lymphadenopathy of intrathoracic lymph nodes (all 1 in number).

Finally, group 5 comprised 23 patients with DR signs of skeletal chest lesions $(n=10 ; 43.5 \%)$, urgent conditions $(n=7 ; 30.4 \%)$ or the clinical picture of lesions was well marked $(n=6 ; 26.1 \%)$.

According to TS results, the changes were confirmed in $91.3 \%$ of cases. Rib fractures unvisualized before were detected in 2 persons (Figure 6). In TS it was difficult to visualize rib fractures on the midaxillary line, probably, due to marginal reduction of spatial resolution in this area. Thus, in one case the lesions were better defined on radiographs in additional oblique projections than in tomograms.

TS additionally revealed fibrotic lung changes $(n=5)$, the signs of subcutaneous emphysema $(n=2)$, hydrothorax and consolidated rib fractures $(n=1)$.

In 9 cases $(39.1 \%)$ the signs of dynamic blurriness due to breathing artefacts were noted on digital tomograms and this reduced visualization of pathological changes.

MSCT performed on three patients allowed hypoventilatory changes, paraseptal emphysema $(n=1)$ and lymphadenopathy of intrathoracic lymph nodes $(n=2)$ to be additionally detected.

Thus, the results of studying the patients with various X-ray semiotic sings have convincingly showed that TS enhances the abilities of $X$-ray radiography. It is superior to $D R$ in the detection of many $X$-ray semiotic signs and can be used as a clarifying examination technique after chest DR to provide better diagnostic accuracy and, in certain cases, to avoid chest MSCT. Nevertheless, MSCT remains the gold standard in diagnosing chest diseases.

Conclusion. Tomosynthesis is inferior to MSCT in the ability to diagnose chest diseases and injuries, however, in $67.2 \%$ of observations it has provided the possibility to obtain additional information which in $39.8 \%$ of cases has proved to be clinically significant and allowed further management of patients to be improved. As a clarifying method, tomosynthesis is able to extend examination algorithm for patients with thoracic pathology.

The presence of marked dyspnea in a patient is a relative counter-indication to perform tomosynthesis.

Study Funding and Conflicts of Interest. This study was not supported by any financial sources, and there is no conflicts of interest associated with this study.

\section{References}

1. Rosstat. Zdravookhranenie v Rossii 2013 [Rosstat. Healthcare in Russia in 2013]. Moscow; 2013.

2. Churkin S.A., Dolgopolov V.B., Temnikov A.A. Problems of the differential diagnostics of pneumonia of the elderly and senility aged persons (on materials of Ftiziogeriatric Department, Orenburg State Financed Health Institution "Tuberculosis Dispensary"). Ftiziatriya i pul'monologiya 2014; 8(1): 50-53.

3. Quaia E., Baratella E., Poillucci G., Kus S., Cioffi V., Cova M.A. Digital tomosynthesis as a problem-solving imaging technique to confirm or exclude potential thoracic lesions based on chest X-ray radiography. Acad Radiol 2013; 20(5): 546-553, http://dx.doi.org/10.1016/j.acra.2012.12.009.

4. Johnsson A.A., Vikgren J., Bath M. Chest tomosynthesis: technical and clinical perspectives. Semin Respir Crit Care Med 2014; 35(1): 17-26, http://dx.doi. org/10.1055/s-0033-1363448.

5. Galea A., Durran A., Adlan T., Gay D., Riordan R., Dubbins P. Williams M.P. Practical applications of digital tomosynthesis of the chest. Clin Radiol 2014; 69(4): 424-430, http:// dx.doi.org/10.1016/j.crad.2013.10.019. 